association between this and high clothing levels $(\mathrm{p}<0.01)$ and gender culture $(\mathrm{p}<0.01)$.

Conclusions CDD reports are associated with sport characteristics such as team structure, contact level, and presence of subjective judging. These may represent true risk factors for misconduct or a stronger culture of reporting. Implications for intentional injury (abuse) prevention are clear.

\section{THE ASSOCIATION BETWEEN PRESEASON FITNESS LEVEL AND RISK OF INJURY OR ILLNESS - A PROSPECTIVE COHORT STUDY IN MALE ELITE ICE HOCKEY}

\begin{abstract}
${ }^{1,2}$ Anine Nordstrøm, ${ }^{1}$ Roald Bahr, ${ }^{1,3}$ Ben Clarsen, ${ }^{2,4}$ Ove Talsnes. ${ }^{1}$ Department of Sports Medicine, Oslo Sports Trauma Research Center, NO, Oslo, Norway; ${ }^{2}$ Innlandet Hospital Trust, Elverum, Norway; ${ }^{3}$ Disease Burden Norwegian Institute of Public Health, Bergen, Norway; ${ }^{4}$ University of Oslo, Oslo University Hospital, Oslo, Norway
\end{abstract}

10.1136/bjsports-2021-IOC.362

Background Little is known about the association between physical fitness and the risk of injury or illness in ice hockey. Objective The aim of this study was to examine the association between pre-season fitness level and injury or illness risk among elite ice hockey players during the subsequent season.

Design Prospective cohort study.

Setting The GET League (the premier professional league in Norway).

Participants Male ice hockey players $(n=133)$.

Interventions (or Assessment of Risk Factors) The players reported all health problems, acute injuries, overuse injuries and illnesses, using the Oslo Sports Trauma Research Center Questionnaire on Health Problems weekly during the 2017/ 2018 competitive season (31 weeks), and completed eight different exercises (40 m sprint, countermovement jump, $3000 \mathrm{~m}$ run, squat, bench press, chins, brutal bench, and box jump) at the annual one-day, pre-season testing combine.

Main Outcome Measurements Number and severity of acute injuries, overuse injuries and illnesses.

Results The number of any health problem did not differ between fitness rank groups, except for all substantial health problems. There was no association between low physical fitness and the number of health problems, comparing the least fit tercile of the players to the rest of the cohort. The least fit players reported a greater cumulative burden of health problems, after adjusting for time on ice per game, position on ice and age.

Conclusions We found no association between low physical fitness and the number of health problems, comparing the least fit tercile of the players to the rest of the cohort. The least fit players reported a greater cumulative burden of health problems, after adjusting for time on ice per game, position on ice and age. Future research focusing on physical performance as risk factors for injury could consider including more specific performance tests on ice.

\section{ARE IMUS SUFFICIENTLY ACCURATE TO MEASURE CHANGES IN 3D KNEE ANGLES AND VELOCITIES DURING THE 70 MS WEIGHT ACCEPTANCE PHASE OF A JUMP LANDING?}

${ }^{1}$ So Young Baek, ${ }^{2}$ Mirel Ajdaroski, ${ }^{1}$ Payam Mirshams Shahshahani, 'Mélanie Beaulieu, ${ }^{2}$ Amanda Esquivel, 'James Ashton-Miller. 'University of Michigan, Ann Arbor, USA; ${ }^{2}$ University of Michigan, Dearborn, USA

\subsection{6/bjsports-2021-IOC.363}

Background The accurate tracking of knee joint motions during maneuvers associated with non-contact ACL injury is important for identifying injury mechanisms.

Objective We tested the hypothesis that motion capture and inertial measurement unit (IMU) measures of 3D changes in tibiofemoral angle and velocity are interchangeable in the $\sim 70$ ms weight acceptance phase of a 3-4*BW dynamic jump landing movement involving knee flexion and tibial internal rotation.

Design Two APDM IMUs and NDI Certus marker triads were rigidly attached to the mid-tibial and -femoral bone of cadaver knees to record motions during simulated jump landings. The initial knee angle was 15 degrees maintained by preparatory quadriceps muscle forces and tensile stiffness for the landing. The Bland-Altman Limits of Agreement (LoA) was used to compare the $3 \mathrm{D}$ data from 852 trials.

Setting University biomechanics research laboratory.

Patients Nine cadaveric knees harvested from six male and three female adult human donors.

Main Outcome Measurements The 3D knee angle changes from motion capture system were considered the gold standard and compared to calculated IMU data from the fusion algorithm provided by APDM Opal. The 3D tibial and femoral angular velocity changes measured by the IMUs were considered the gold standard and compared to the differentiated Certus angular data.

Results Although the mean peak IMU knee angle changes were slightly underestimated in all three orthogonal planes, the LoA bands were large, ranging from $35.9 \%$ to $49.8 \%$. Certus had acceptable accuracy in the camera plane for angular velocity changes, with LoAs of $\pm 54.9 \%$ sec and $\pm 32.5 \%$ sec, respectively, for the tibia and femur.

Conclusions These IMUs could not reliably measure the peak 3D knee angle changes. Certus measurements of tibiofemoral angular velocity changes were comparable to IMU measures in the camera plane, and when velocities were sufficiently large.

\section{EPIDEMIOLOGY OF NON-CONTACT ACL INJURIES IN TEAM BALL-SPORTS: A SYSTEMATIC REVIEW WITH META-ANALYSIS AND META-REGRESSION OF 2748 INJURIES ACROSS 42 MILLION PLAYER-HOURS AND - EXPOSURES COMBINED}

${ }^{1}$ Lionel Chia, ${ }^{2}$ Danilo De Oliveira Silva, ${ }^{3}$ Matthew Whalan, ${ }^{4}$ Fuller Colin, ${ }^{1}$ Marnee McKay, ${ }^{1}$ Justin Sullivan, ${ }^{1,5}$ Pappas Evangelos. ${ }^{1}$ The University of Sydney, NSW, Australia; ${ }^{2}$ La Trobe University, NSW, Australia; ${ }^{3}$ Football Australia, NSW, Australia; ${ }^{4}$ Colin Fuller Consultancy, NOTT, UK; ${ }^{5}$ University of Wollongong, NSW, Australia

10.1136/bjsports-2021-IOC.364

Background Not all ACL injuries are preventable. The epidemiology of non-contact ACL injuries is not known. 